\title{
The genetics of cornea plana congenita
}

Esa Tahvanainen, Henrik Forsius, Juha Kolehmainen, Margareta Damsten, Johan Fellman, Albert de la Chapelle

\begin{abstract}
Cornea plana congenita is believed to occur in a mild autosomal dominant (CNA1) and a more severe autosomal recessive (CNA2) form. We recently assigned a CNA2 locus to a region on chromosome 12 by linkage analysis. In this study we compared these traits clinically and genetically. Using the horizontal corneal refraction value in diopters (D) as a parameter, a control population $(n=473)$ had a mean value of 43.4 (SD $1.5 \mathrm{D}$ ) for men and 43.7 (SD 1.6 D) for women, whereas in 51 subjects affected with CNA2 the mean value was 29.9 (SD 5.2 D) and in five subjects affected with CNA1 the mean value was 37.8 (SD 1.6 D). By linkage analysis in two CNA1 families the CNA2 locus could be conclusively excluded. These data suggest that at least two forms of hereditary cornea plana exist which are both clinically and genetically distinct.

( $($ Med Genet 1996;33:116-119)
\end{abstract}

Key words: cornea plana congenita; linkage study.

In cornea plana the normal protrusion of the cornea from the sclera is missing. The existence of heritable forms of cornea plana congenita has long been known; however, in the earliest published studies a clear demarcation from other corneal malformations was difficult. ${ }^{1-4}$ More recently a classification based on inheritance patterns and clinical signs has been proposed..$^{5-7}$ Presently McKusick's catalog ${ }^{8}$ lists an autosomal dominant form (CNA1, MIM 121400) and an autosomal recessive form (CNA2, 217300). In addition cornea plana can occur as a part of more severe ocular malformations. An entity called autosomal dominant peripheral sclerocornea (MIM 181700), in which the cornea is flatter than normal is difficult to distinguish from CNA1, while a severe form of sclerocornea may exhibit recessive inheritance ${ }^{10}$ (MIM 269400).

The dominant and recessive forms of cornea plana share many clinical features, including reduced corneal curvature in most cases leading to hyperopia, hazy corneal limbus, and arcus lipoides at an early age. The differential diagnosis is partly based on the more severe symptoms of the recessive form. In particular, a round and opaque thickening located centrally in the cornea with a diameter of about $5 \mathrm{~mm}$ is the most typical finding associated with recessive cornea plana. In the recessive form additional anomalies are often present, such as malformations of the iris, a slit-like pupil, and adhesions between the iris and cornea.
Recently linkage analysis with polymorphic markers allowed us to map the locus for CNA2 in Finnish families. ${ }^{11}$ It resides in a $3 \mathrm{cM}$ interval flanked by markers D12S82 and D12S327 in the proximal part of the long arm of chromosome 12. In this paper we examine two aspects of cornea plana. First, can people with the putative dominant and recessive forms be distinguished from each other and from normal subjects by studying the corneal refraction? Second, are the two forms genetically distinct?

\section{Materials and methods}

CONTROL POPULATION

The people in the archipelago of Kökar which belongs to the Åland Islands situated off the south west coast of Finland form an isolated population where genealogical studies and repeated measurements are easy to perform. We performed an ophthalmological clinical field study of consenting subjects on two occasions, in 1960-1962 and in 1990-1992. Most people were studied on both occasions. The study population consisted of 243 normal males and 230 normal females without significant ophthalmological disease. For a detailed description of the ophthalmological features of the control population readers are referred to previous papers. ${ }^{1213}$ Because of the possible effects of young age on the refraction power, children under school age were not included in the current study.

\section{DOMINANTLY INHERITED CORNEA PLANA} CONGENITA

Over a 35 year period, we have diagnosed a condition compatible with dominant cornea plana in only two families, comprising seven affected subjects. While the affected people live in different parts of Finland, the genealogies of both families were traceable to a rural community in west central Finland as previously reported. ${ }^{6}$ The mendelian nature of the inheritance in these families is sustained by the fact that they have shared ancestry (fig 1). The patients showed the typical signs of dominant cornea plana, that is, indistinct border of the sclera and cornea without any notable angle, arcus lipoides at an early age, and lowered anterior chamber depth. Horizontal corneal refraction values were measured in five of the six affected adults (data on the youngest child who was 4 years of age at the time of study were not included). The values were as follows: the single affected child in the family on the left (fig 1) $39 / 40$ (right/left eye), the mother and oldest three children in the family on the right $38 \cdot 75 / 39 \cdot 5,37 / 37 \cdot 5,34 \cdot 5 / 37 \cdot 5$, and 


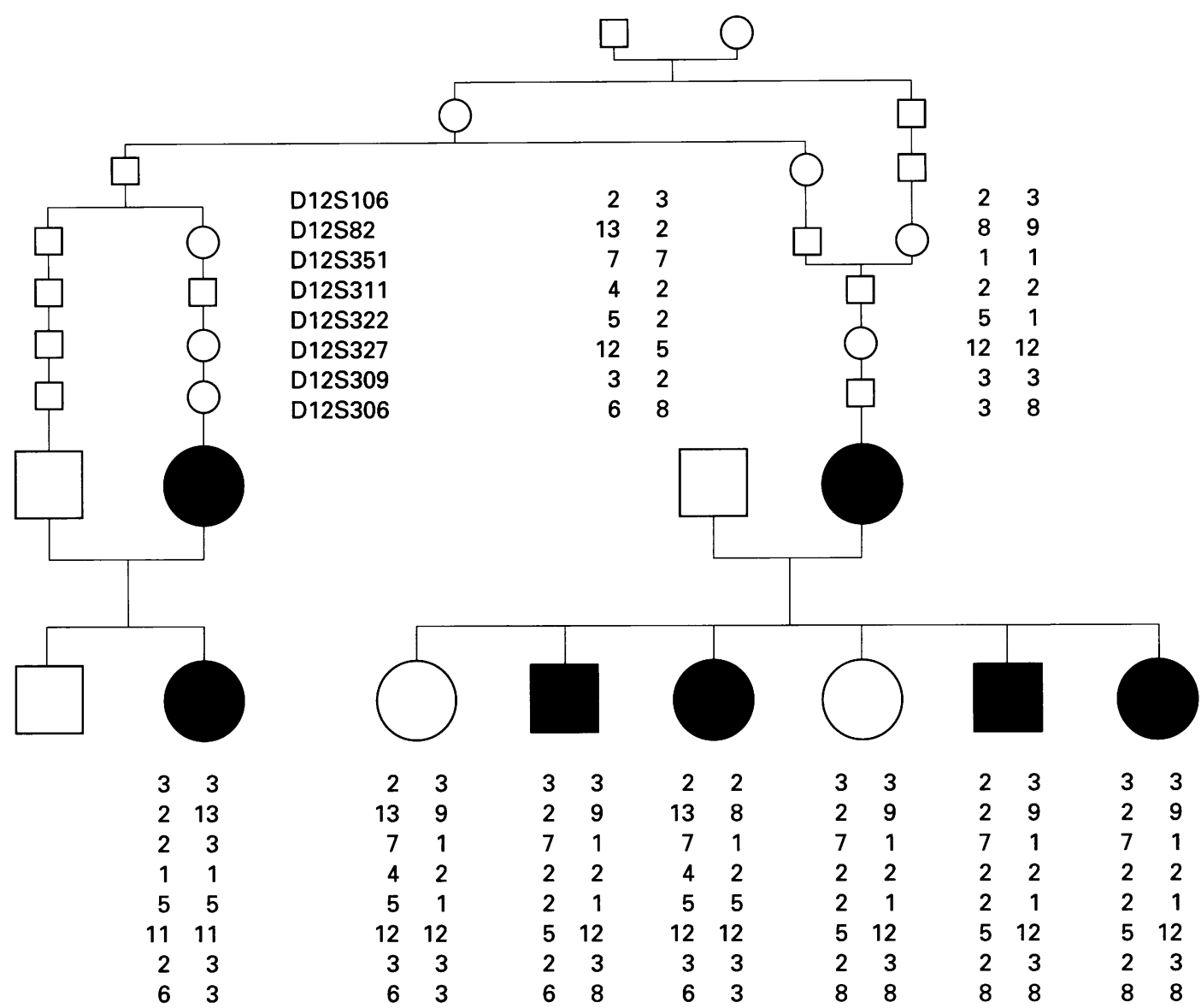

Figure 1 Pedigree of two cornea plana families showing dominant inheritance. The suggested haplotype arrangement was either derived directly from parental alleles or based on the lowest number of recombinations. The allele arrangement in the affected child on the left does not imply haplotyping.

$36 \cdot 75 / 37$. Central opacity of the cornea typical of the recessive form was not observed, nor any other malformation except for a minor iris anomaly in one eye. Glaucoma was not observed. One 50 year old woman had a mature cataract in one eye.

\section{RECESSIVELY INHERITED CORNEA PLANA \\ CONGENITA}

This phenotype occurs with relatively high incidence in the isolated population of northern Finland, where altogether about 90 affected subjects have been diagnosed. In the region of the lower parts of the Kemijoki river, as many as 26 patients are known to have common ancestry dating approximately 10 to 15 generations back as shown by genealogical data. The clinical and genealogical features and diagnostic criteria of this disease have been published, ${ }^{56}$ as well as the recent assignment of its gene locus (CNA2) to chromosome 12 by linkage. ${ }^{11}$ Corneal refraction values reported here are from the right eye of 51 subjects, most of whom are newly diagnosed cases.

\section{ASSESSMENT OF CORNEAL STATUS}

In addition to careful inspection the investigation included measurement of the corneal radius with the Javal-Schiötz ophthalmometer (Haag-Streit). The axis nearest to the horizontal plane was taken as the horizontal value. The values are given in diopters (D). The corneal radius in $\mathrm{mm}$ can be calculated from the formula $r=336 / \mathrm{D}$.

\section{LINKAGE AND HAPLOTYPE ANALYSIS}

Linkage and haplotype analyses were based on eight polymorphic markers (D12S106, D12S82, D12S351, D12S311, D12S322, D12S327, D12S309, D12S306) around the recessive cornea plana gene locus. All marker data, including order and distance, have been published. ${ }^{14}$ The polymerase chain reactions (PCR) were done in $10 \mu \mathrm{l}$ reaction volumes using published protocols. ${ }^{15}$ The LINKAGE program package ${ }^{16}$ was used for the linkage analyses. A LINKMAP analysis was carried out against a fixed map of four informative marker loci in the order centromere-D12S106D12S82-D12S322-D12S306-telomere with distances of $4 \mathrm{cM}, 2 \mathrm{cM}$, and $9 \mathrm{cM}$ between the markers. Haplotypes were constructed based on a minimum number of recombinations for those loci in which the haplotypes could not be determined by studying the parents (fig 1).

\section{Results}

HORIZONTAL CORNEAL REFRACTION VALUES

The mean horizontal corneal refraction value measured from the right eye of 473 ophthalmologically healthy Kökar islanders, who 


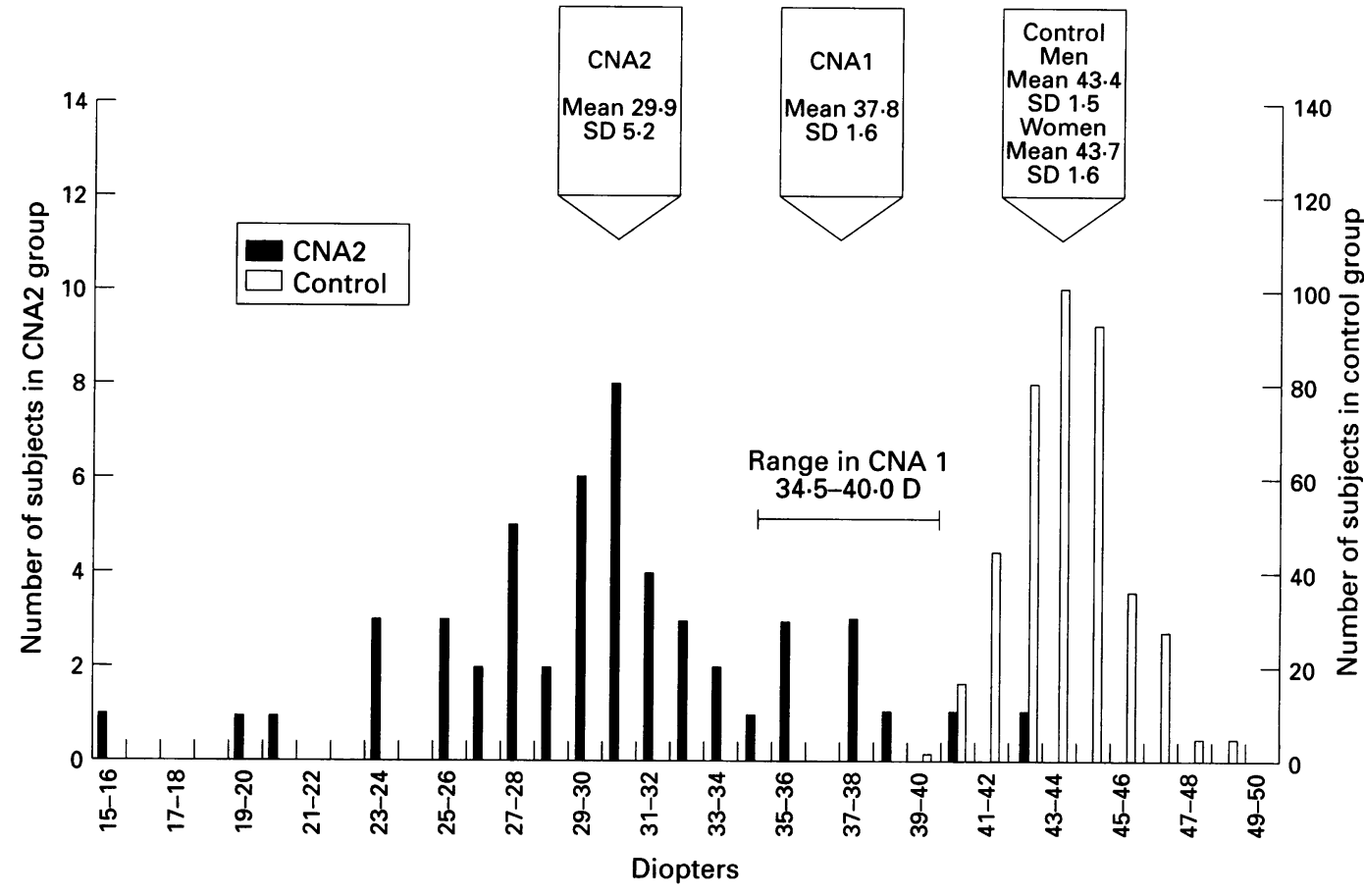

Figure 2 Individual horizontal corneal refraction values and their means measured from the right eye of 51 CNA2 patients and 473 controls. The mean and range of the values measured from both eyes of five CNA1 patients is also shown.

represent the control population in this study (fig 2), was 43.4 (SD 1.5 diopters) for men and 43.7 (SD 1.6 diopters) for women. Horizontal corneal refraction values of the right eye of 51 affected subjects belonging to CNA2 families showed a range of corneal refraction values of $15 \cdot 0-42 \cdot 75 \mathrm{D}$, with the overall mean $29 \cdot 5 \mathrm{D}$ (SD $5 \cdot 2 \mathrm{D})$. We obtained a kurtosis value of 0.52 and skewness value of -0.18 for the distribution in CNA2 and $0 \cdot 11$ and $0 \cdot 28$ (men) and 0.00 and 0.26 (women) in the control population, respectively. Based on these numerical kurtosis values the distribution of refraction values in CNA2 is more centred around its mean value $(29.9 \mathrm{D})$ than in the controls.

Thus, owing to greater variance, the CNA2 distribution is only apparently flatter (fig 2).

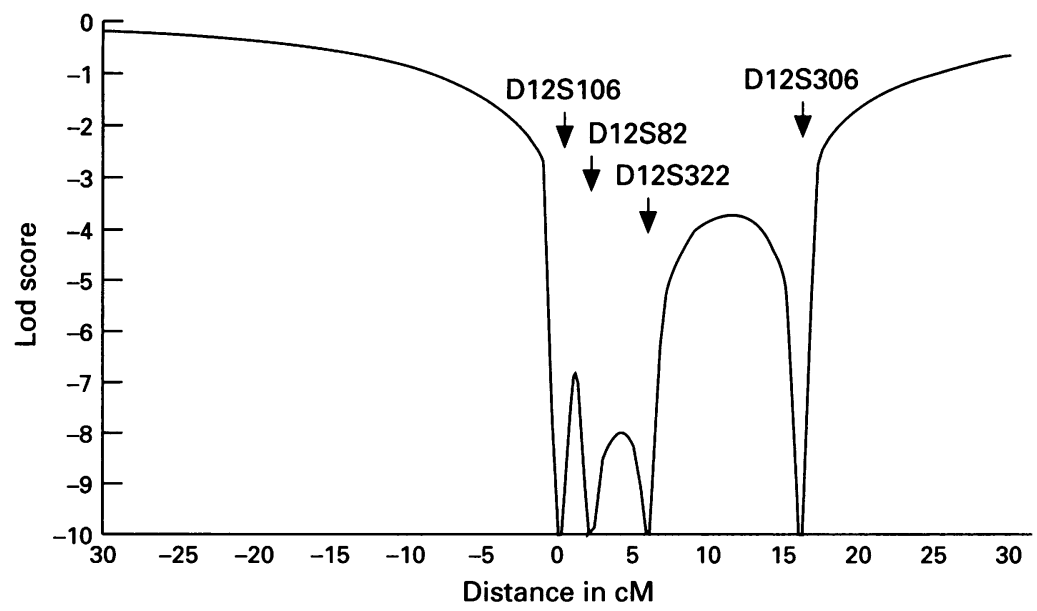

Figure 3 Five point linkage analysis in two CNA1 families with respect to four marker loci on chromosome 12 computed using the LINKMAP program. Marker D12S106 was chosen as the starting point (zero). Sex average distances based on Haldane mapping function are shown. The centromere is to the left.
The range of corneal refraction values measured in both eyes of five affected subjects belonging to the CNAl pedigree (fig 1) was $34 \cdot 5-40 \mathrm{D}$ (fig 2), the mean was $37 \cdot 8 \mathrm{D}$, and the standard deviation $1.6 \mathrm{D}$ (mean and standard deviation calculated from both eyes because of the small number of observations).

\section{LINKAGE STUDY}

The alleles shown (fig 1) are arranged as most likely haplotypes. It can readily be seen that linkage of the cornea plana phenotype to the chromosomal region under study is highly unlikely. By pairwise linkage analysis (table) all informative markers, D12S106, D12S82, D12S322, and D12S306, showed negative lod scores with the CNA1 phenotype and gave exclusions (lod scores below -2) extending 1 to $5 \mathrm{cM}$ from the markers. Markers for which the mother was homozygous were uninformative, as we did not take the connection between the two families into account in the linkage analysis. By multipoint analysis the whole area was excluded by the criterion of a lod score below -2 (fig 3 ). The above results were calculated assuming dominant inheritance with recombination fractions from each family branch added together. Assuming recessive inheritance instead, the region is also excluded (data not shown).

\section{Discussion}

One of our goals was to assess the value of corneal refraction power measurements in distinguishing these two disorders and the control population from each other. To this end we first studied a control population of 473 subjects in whom the refraction values did not differ from 
Pairwise lod scores between CNA1 and four marker loci

\begin{tabular}{llllllll}
\hline Locus & 0.00 & 0.001 & 0.01 & 0.05 & $0 \cdot 10$ & 0.20 & 0.30 \\
\hline D12S106 & $-\infty$ & -4.80 & -2.80 & -1.44 & -0.89 & -0.39 & -0.15 \\
D12S82 & $-\infty$ & -7.20 & -4.21 & -2.16 & -1.33 & -0.58 & -0.23 \\
D12S322 & $-\infty$ & -7.20 & -4.21 & -2.16 & -1.33 & -0.58 & -0.23 \\
D12S306 & $-\infty$ & -4.50 & -2.51 & -1.18 & -0.67 & -0.25 & -0.09 \\
\hline
\end{tabular}

a Gaussian distribution curve that may be best explained by multifactorial determination with a clear cut hereditary component. The effects of putative genes on the trait are illustrated by correlations between parents and children that we showed in a previous study of the same population. ${ }^{5}$ Mother-daughter correlations were clear cut (regression coefficient 0.512 , $\mathrm{p}<0.001)$ and father-daughter correlations lower $(0.252, \mathrm{p}<0.05) .^{5}$

The values in CNA2 patients showed considerable variation; however, as a group, they were dramatically lower than in the controls. Only two out of 51 patients had values that overlapped with the lowest control values; this suggests that the refraction value should not be used as the sole diagnostic criterion. Clinically this is no problem as CNA2 is almost always associated with other manifestations of ocular disease (see Introduction). The mean refraction value obtained from five CNA1 patients fell between those of CNA2 patients and the controls. This is in line with results from two other studies. ${ }^{34}$ Altogether these data suggest that in addition to pedigree and clinical data, the measurement of horizontal corneal refraction helps distinguish between controls and cornea plana patients, and that the dominantly and recessively inherited forms differ from each other not only in terms of mode of inheritance and clinical signs, but also in corneal refractive power.

The linkage study we performed in two families with dominantly inherited cornea plana clearly shows that the trait is not caused by mutations at the CNA2 locus on chromosome 12. This establishes the genetic, in addition to the clinical, heterogeneity of the two conditions. If, contrary to the most likely interpretation, these families display recessive inheritance, the CNA2 locus is excluded as well. For the sake of completeness it should perhaps be mentioned that neither $\mathrm{X}$ chromosomal dominant nor mitochondrial in- heritance can be formally excluded either. In any case, our finding obviously applies only to the two families studied by us. Other cornea plana families with dominant inheritance has been studied in the USA, ${ }^{3}$ Denmark, ${ }^{4}$ Germany, ${ }^{7}$ and Netherlands. ${ }^{17}$ Until the underlying genes have been mapped and cloned it will not be possible to assess the degree of genetic heterogeneity that they may display.

We thank Sinikka Lindh for collecting the samples, Verna Carlsson for pedigree analysis, Seppo Sarna for advice on the statistics, and the cornea plana families for their cooperation. This study was supported by the Academy of Finland, the Paulo Foundation, The Finnish Medical Foundation, and the Finnish Foundation for Pediatric Research, Ulla Hjelt fund.

1 Rübel B. Kongenitale familiäre Flachheit der Kornea. Klin Monatsbl Augenheilkd 1912;50:427-33.

2 Felix $\mathrm{CH}$. Kongenitale familiäre Cornea plana. Klin Monatsbl Augenheilkd 1925;74:710-16.

3 Barkan H, Borley WE. Familial cornea plana complicated by cataracta nigra and glaucoma. Am $\mathcal{f}$ Ophthalmol 1936; 19:307-10.

4 Larsen V, Eriksen A. Cornea plana. Acta Ophthalmol (Copenh) 1949;27:275-86.

5 Forsius $\mathrm{H}$, von Fieandt $\mathrm{O}$. Photokeratometry and refraction analysis in congenital cornea plana. Acta Ophthalmol (Copenh) 1963;41:609-20

6 Eriksson AW, Lehmann W, Forsius H. Congenital cornea plana in Finland. Clin Genet 1973;4:301-10.

7 Ruprecht KW, Naumann G. Perforierende Keratoplastik und Histopathologie der Cornea plana. Klin Monatsbl Augenheilkd 1974;165:585-94.

8 McKusick VA. Mendelian inheritance in man. 11th ed. Baltimore: Johns Hopkins University Press, 1994.

9 Elliot JH, Feman SS, O'Day DM, Garber M. Hereditary sclerocornea. Arch Ophthalmol 1985;103:676-9.

10 Bloch N. Les differents types de sclerocornee, leurs modes d'hérédité, et les malformations congenitales cond'heredite, et les malformations congent

11 Tahvanainen E, Forsius H, Karila E, et al. Cornea plana congenita gene assigned to the long arm of chromosome 12 by linkage analysis. Genomics $1995 ; 26: 290-3$.

12 Forsius H, Eriksson AW, Fellman J. Corneal refraction according to age and sex in an isolated population and the heredity of the trait. Acta Ophthalmol (Copenh) 1964 42:244-35.

13 Forsius H, Eriksson AW, Fellman J. A fishing population in Kökar/Åland. State of their eyes in 1960-62 and 1991 Arch Public Health 1994;51:549-60.

14 Gyapay G, Morissette J, Vignal A, et al. The 1993-94 Généthon human genetic linkage map. Nature Genet 1994; 7:246-339.

15 Weber JL, May PE. Abundant class of human DNA polymorphisms which can be typed using the polymerase chain morphisms which can be typed using the poly

16 Lathrop GM, Lalouel JM, Julier C, Ott J. Strategies for multilocus linkage analysis in humans. Proc Natl Acad Sci USA 1984;81:3443-6.

17 Waardenburg PJ. Hornhautrefraktion und Hornhautscheibengrösse. Klin Monatsbl Augenheilkd 1930;85:169 185. 